\title{
CFD-Exergy analysis of the flow in a small-sized Venturi
}

\author{
Ahmed Razali ${ }^{1}$, Mohammed Baghdad $^{2}$, and Ahmed Ouadha ${ }^{1, *}$ \\ ${ }^{1}$ Laboratoire des Sciences et Ingénierie Maritimes, Faculté de Génie Mécanique, Université des Sciences et de la Technologie \\ Mohamed Boudiaf d'Oran, Oran El-M'nouar, 31000 Oran, Algérie \\ ${ }^{2}$ Institut des Sciences et de la Technologie, Centre Universitaire El-Wancharissi, B.P. 182, 38000 Tissemsilt, Algérie
}

\begin{abstract}
The present study aims to compare cavitation models in predicting the flow in small-sized cavitating venturis. Three cavitation models, namely Schnerr and Sauer model, Zwart et al. Model and Singhal et al. Model have been compared under the mixture approach. Furthermore, the performance of this device has been assessed using the concept of exergy by quantifying the exergy losses accuring in its different parts. It is found that all models are capable of reproducing the physics of cavitation phenomena within the cavitating venturi. However, the Schnerr and Sauer model return higher values than the others model. It is also observed that most of the exergy losses occur in the converging and diverging parts of the venturi due to higher pressure and velocity gradients in these regions.
\end{abstract}

Keywords. Small sized venturi tube; cavitation; CFD; exergy analysis

\section{Introduction}

A venturi tube is a low-pressure loss device which consists of three sequential parts: a convergent section, a throat and a divergent section. Due to their simple structure, venturi tubes are widely encountered in several industrial applications, including food processing, oil and gas industry, transportation vessels, agriculture, ... etc. They are considered as a mature technology that can be used for a wide range of liquids, gases and steam. Furthermore, they are also suitable for passage of multiphase flows and wet gases.

Venturi tubes can operate under non-cavitating or cavitating modes. Under the cavitating mode, the minimum pressure is reduced to the fluide vapor pressure at the corresponding temperature. Cavitating venturis are mainly used in flow limiters, mixture ratio controllers, injectors, actuator movement equalizers and fire extinguishers. As flow control devices, they allow a liquid flow rate to be fixed or locked independently of the downstream process conditions or fluctuations.

Due to their simplicity, cavitating venturis have been widely investigated from both numerical and experimental approaches. While earlier works were exclusively experimental and focused on theoretical bases of cavitating venturis and their sizing features [18], the development of CFD codes has accelerated the investigation of cavitating venturis using several numerical models. Later, Sherwood [9] has reported computations of the pressure fluctuation due to the passage of a single bubble. The liquid is assumed inviscid and its velocity, assumed irrotational, is computed by means of a boundary integral technique.
Gaston et al. [10] have developed a simple model for cavitation bubble dynamics using a combination of boundary element methods and one-dimensional bubble dynamic equations. Park et al. [11] proposed an improved propellant feed system for the stable combustion of liquid rocket. This system utilizes the cavitating venturi to provide stable mass flow rate. The results showed that the cavitating venturi effectiveness increased when the ratio of actual feed pressure to the cavitating venturi design pressure is increased. It is also found that Kerosene is more effective to supply stable mass flow rate than LOx. Sato et al. [12] have observed the whole process of generation, development and collapse of the cavitation bubbles. They found that a traveling-bubble cavitation appears dominantly in a nozzle-type venturi tube with little possibility of flow separation. Cavitation aspects and the bubble occurrence count rate change with cavitation number and water quality (dissolved gas content). Unstable sheet cavitation can be also observed near the venturi diffuser and at relatively low cavitation number. Palau-Salvador and Frankel [13] have compared numerical predictions of cavitation in a series of orifice and nozzle flows to experimental measurements as means of validating the new cavitation model in the commercial CFD code Fluent. Kang et al. [14] have investigated the flow rate control of a cavitating venturi with downstream pressure variation. Their results showed that constant mass flow rate condition was established by the cavitation inside the venturi. However, upstream pressure less than the actual design pressure of the cavitating venturi could not supply a constant flow rate. Yazici et al. [15] have investigated numerically and experimentally venturi

\footnotetext{
Corresponding author: ah ouadha@yahoo.fr
} 
flows. They showed that the cavitation bubbles with high momentum would collapse to the cavitation bubbles with low momentum. An and Plesniak [16] have studied experimentally cavitation inception and development caused by a backward facing step in a venturi-type test section for different step heights $(0.8-4 \mathrm{~mm})$. Highspeed cinematography and particle image velocimetry images were used to demonstrate the cloud shedding process and cavitation development stages. It was found that for small step height $\left(0.8 \mathrm{~mm}, h \delta_{0}=1.7\right)$ cavitation started in the reattachment region in the form of a reentrant jet, and caused cloud shedding in its developed stage. For high step height $(4 \mathrm{~mm}, \mathrm{~h} \delta 0=8.5)$ cavitation started midway downstream of the shear layer and resembled the spanwise vortices in the separated shear layer. Barre et al. [17] have carried out correlated experimental and numerical studies to analyze cavitating flows and to describe the two-phase flow structures of attached sheet cavitation in Venturi geometries. They estimated void ratio and velocity fields for cold water flows using new double optical probe measurements and special data processing methods. Sayyaadi [18] has experimentally investigated the instability characteristics of cavitating flow in a venturi tube in a recirculating flow loop in order to observe the fluctuation process of cavitation flow using high-speed photography. He showed that cavitation number has a major effect on the fluctuation characteristics of the cavitating flow in comparison with the effect of operating pressure. It was observed also that at lower cavitation numbers, periodic separation, shedding and collapsing of the cloud-like cavities are observed together with the re-entrant jet motion. At higher cavitation numbers, the simple fluctuation mode with a sinusoidal pattern is observed. Ghassemi and Fasih [19] have designed and built three cavitating venturis with throat diameter of 5, 2.5, and $1 \mathrm{~mm}$ to investigate the effect of venturi size on its mass flow rate. They found that for the ratio of downstream pressure to upstream pressure less than 0.8 , the mass flow rate is constant and independent of the downstream pressure. The venturi acts like an orifice for pressure ratios greater than 0.8 . Decaix and Goncalvès [20] have performed a numerical investigation of the behavior of a cavitation pocket developing along a Venturi geometry using a compressible one-fluid hybrid RANS/LES solver. Abdulaziz [21] has conducted experiments on a small type venturi test rig under varying upstream, throat and downstream conditions. He proposed and validated also a model for water vapor void fraction. Experimental and model results showed that cavitation occurs at a certain critical pressure ratio (downstream pressure/upstream pressure) where the liquid starts to evaporate at the throat of the venturi. Rodio and Congedo [22] have analyzed the impact of various sources of uncertainty on the prediction of cavitating flows by coupling a non-intrusive stochastic method with a cavitating CFD solver. They proposed also a simple algorithm in order to provide an optimized set of parameters for the cavitation model. Tian et al. [23] have built and tested a variable area cavitating venturi to examine the mass flow rates different pintle positions, upstream pressures, downstream pressures and dynamic motions of the pintle. They found that the mass flow rate is independent of the downstream pressure when the ratio of the downstream pressure to upstream pressure is less than 0.8. The mass flow rate is almost linearly dependent on the pintle stroke for a constant upstream pressure. The discharge coefficient is a function of the pintle stroke, whereas the upstream and downstream pressures have rare influence on the discharge coefficient. Charrière et al. [24] presented a numerical study of an aperiodic cavitation pocket developing in a venturi flow. They proposed and compared a new freeparameter closure relation with other formulations. Mena et al. [25] have detailed the design and performance characterization for a cryogenic cavitating venturi. The test results revealed that the cavitating venturi indeed performed as a flow rate control feature in both liquid water and LCH4 flow under a steady state operating within pressure ratios below 0.69. Ashrafizadeh and Ghassemi [26] have experimentally and numerically investigated the effect of upstream and downstream pressures, as well as geometrical parameters such as the throat diameter, throat length, and diffuser angle on the mass flow rate and critical pressure ratio in a small-sized cavitating venturi. They found that despite the small size of venturis, they are capable of controlling the mass flow rate and exhibit the normal characteristics. By decreasing the throat diameter, their cavitating mode became more limited. Results also show that increasing the diffuser angle and throat length leads to a decrease in critical pressure ratio. Long et al. [27] have conducted experiments to investigate the global cavitation behavior in a venturi tube. Their results showed that once cavitation occurs, the flow rate remains almost constant regardless of the outlet pressure variations, and the pressure ratio and cavitation number are linearly related. Cavitation occurs each time regardless of the inlet or outlet pressure at the same critical pressure ratio of 0.89 , which corresponds to a critical cavitation number of 0.99 . The cavity length is only the function of the pressure ratio or the cavitation number independent of the inlet pressures. Zhu et al. [28] experimentally investigated the dynamic cavitation characteristics of liquid nitrogen flow through a transparent venturi tube in a variable pressure ratio tunnel. They found that the shedding frequency and length of cavity linearly increases, while the pressure amplitude exponentially increases, as the pressure ratio increases. They observed also a shock wave induced condensation front propagating upstream within the attached cavity when the pressure ratio approximately exceeds 2.23.

Since its establishment by Bejan [29], entropy generation analysis has received a growing interest in optimization of thermal processes. Entropy generation is directly related to thermodynamic irreversibilities, which characterize any real process. Hence, methods based on entropy generation minimization can be considered as a robust tool in the optimization of thermal processes.

Although a great deal of studies has been dedicated to entropy generation analysis in thermal processes, there is a paucity of studies on the application the second law of thermodynamics to this device. Bulk exergy analysis which is based on only inlet and outlet conditions does 
not take into account the complexity of the flow within a cavitating venturi. Detailed information about the local entropy generation are necessary in order to quantify and locate mechanisms that produce irreversibilities. The objective of this study is to create a user-friendly simulation modelling that can be easily used in the analysis of the cavitation phenomena in a venturi tube using the concept of exergy analysis.

\section{Mathematical Modeling and Solution Method}

\subsection{Physical domain}

Fig. 1 illustrates the computational domain used for the present study. The cavitating venturi tube built and tested by Ashrafizadeh and Ghassemi [26] has been selected for the present study. It consists of convergent section, a throat and a diffuser. The inlet and outlet diameter is $D=4 \mathrm{~mm}$. The throat diameter and length are fixed to $1 \mathrm{~mm}$. Angles of converging and diverging sections have been fixed to 15 and $7^{\circ}$, respectively. In order to obtain steady and fully developed flows at the entry and exit of the venturi, the venturi is extended by two pipes with a length $L_{\text {pipe }}=20 \mathrm{~cm}$ added to both sides. The overall length of the venturi became $40 \mathrm{~mm}$.

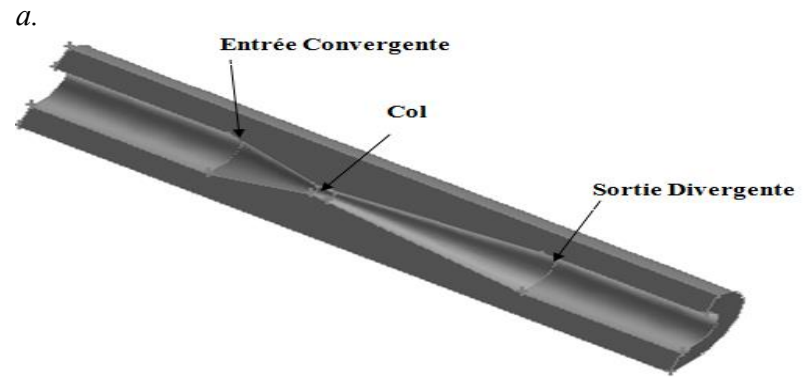

$b$.

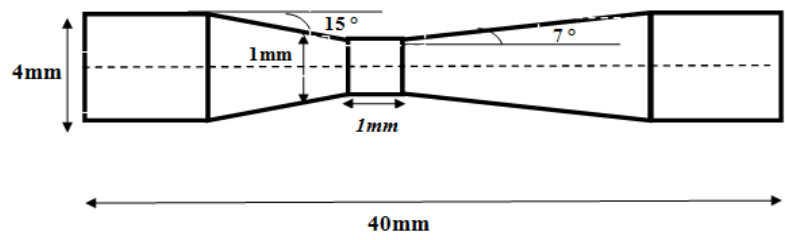

Fig. 1. Description of the venturi studied : $a$. Overall View ; $b$. Dimensions

\subsection{Governing equations and boundary conditions}

Several numerical models have been used to investigate cavitating flows. Among these models, the homogeneous mixture model treats the cavitating flows as a mixture of two fluids behaving as one. It is considered as relatively simple model that assumes a local kinematic equilibrium between phases and a local thermal and mechanic equilibrium between the two components.

Using the mixture approach, the two-phase flow is governed by continuity and momentum equations:

$$
\frac{\partial \rho}{\partial t}+\frac{\partial\left(u_{j} \rho\right)}{\partial x_{j}}=0
$$

$$
\begin{aligned}
& \frac{\partial\left(\rho u_{i}\right)}{\partial t}+\frac{\partial\left(\rho u_{i} u_{j}\right)}{\partial x_{j}}=-\frac{\partial p}{\partial x_{i}} \\
& +\frac{\partial}{\partial x_{j}}\left[\mu_{e f f}\left(\frac{\partial u_{i}}{\partial x_{j}}+\frac{\partial u_{j}}{\partial x_{i}}-\frac{2}{3} \frac{\partial u_{i}}{\partial x_{i}}\right)\right]
\end{aligned}
$$

where the mixture density, $\rho$, is calculated using

$$
\frac{1}{\rho}=\frac{f_{v}}{\rho_{v}}+\frac{1-f_{v}}{\rho_{l}}
$$

with $\rho_{v}$ and $\rho_{l}$ denote for vapor and liquid densities, respectively and $f_{v}$ is the vapor mass fraction.

In cavitation, the liquid-vapor mass transfer is governed by the vapor transport equation:

$$
\frac{\partial(\rho \alpha)}{\partial t}+\frac{\partial\left(u_{j} \rho \alpha\right)}{\partial x_{j}}=R_{p}
$$

where $\alpha, \rho_{v}$ and $u$ denote vapor volume fraction, vapor density and vapor phase velocity, respectively. $R_{p}$ is mass transfer source term related to the growth and collapse of the vapor bubbles.

Under the mixture model, Fluent uses three approaches to model the source term governing the vaporization and condensation processes: Schenerr and Sauer model, Zwart-Gerber-Belamri model and Singhal et al. model.

According to Schnerr and Sauer [30], vapour volume fraction is function of bubble radius and bubble number density:

$$
\alpha=\frac{\frac{4}{3} \pi R^{3} \eta}{1+\frac{4}{3} \pi R^{3} \eta}
$$

where $R$ is the bubble radius and $\eta$ is the bubble number density $(\eta=1013 \mathrm{~m}-3)$. The liquid-vapor mass transfer is governed by the vapor transport equation which can be obtained by combining the time derivative of $\alpha$ with the vapor and liquid mass conservation equations:

$$
\frac{\partial(\rho \alpha)}{\partial t}+\frac{\partial\left(u_{j} \rho \alpha\right)}{\partial x_{j}}=R_{p}
$$

The source term, $R_{p}$, is determined using Rayleigh equation by:

$$
R_{p}=\frac{3 \alpha(1-\alpha)}{R} \frac{\rho_{v} \rho_{l}}{\rho}(-1)^{j} \sqrt{\frac{2}{3} \frac{\left(\left|p-p_{v}\right|\right)}{\rho_{l}}}
$$

where,

$$
p-p_{v}\left\{\begin{array}{lll}
>0 & j=1 & \text { condensation } \\
<0 & j=2 & \text { evaporation }
\end{array}\right.
$$

For Zwart et al. [31] Model, the source term, $R_{p}$, is determined assuming a constant bubble size. Non- 
condensable gases have not been taken into account for this model.

For $p<p_{v}$ (evaporation),

$$
R_{p}=F_{\text {vap }} \frac{3 \alpha_{\text {nис }}(1-\alpha) \rho_{v}}{R} \sqrt{\frac{2\left(p-p_{v}\right)}{3 \rho_{l}}}
$$

For $p>p_{v}$ (condensation),

$$
R_{p}=F_{\text {cond }} \frac{3 \alpha \rho_{v}}{R} \sqrt{\frac{2\left(p-p_{v}\right)}{3 \rho_{l}}}
$$

where $\alpha=\alpha_{v} ; F_{\text {vap }}=50 ; F_{\text {cond }}=0.01 ; R=10^{-6}$ is the bubble radius; $\alpha_{n u c}=5 \times 10^{-4}$ is the nucleation site volume fraction.

For Singhal et al. [32] model, the vapor phase includes both vapor and non-condensable gases. The saturation pressure has been corrected by an estimation of the local values of the turbulent pressure fluctuations:

$$
p_{v}=p_{\text {sat }}+\frac{1}{2}(0.39 \rho k)
$$

The fluid density is expressed as a function of gaseous mass fraction $f_{v}$ which requires an additional equation to be coupled with continuity and momentum equations. For each phase, the volume fraction is related to the mass fraction by

$$
\left\{\begin{array}{l}
\alpha_{v}=f_{v} \frac{\rho}{\rho_{v}} \\
\alpha_{l}=f_{l} \frac{\rho}{\rho_{l}}
\end{array}\right.
$$

The empirical constants for evaporation and condensation $f_{\text {vap }}$ and $f_{\text {cond }}$ are fixed to 0.02 and 0.01 , respectively. $\sigma$ is the surface tension of liquid; $k$ is the turbulent kinetic energy; $f_{v}$ and $f_{g}$ are the mass fraction of vapor and non-condensable gases.

Reynolds stresses, terms that arise during the Reynolds averaging of Navier-Stokes equations, have been modeled using the standard $k-\varepsilon$ turbulence model.

As boundary conditions, pressures are fixed at the inlet and outlet of the venturi tube, and no-slip boundary conditions are specified at the wall. Turbulent intensity and hydraulic diameter are also specified at the inlet and outlet of the venturi tube. During experimental tests [26], the inlet pressure has been maintained at $2 \mathrm{MPa}$, while the outlet pressure has been varied from 0.5 to $1 \mathrm{MPa}$. For all cases, the temperature has been fixed to $300 \mathrm{~K}$.

All natural processes are irreversible. The thermodynamic irreversibility in a process is characterized by the entropy generation. Since its establishment by Bejan [26], entropy generation minimization has become a topic of great interest in the thermo-fluid area. In his work cited above, Bejan has combined the concept of fluid flow and heat transfer with the second law of thermodynamics in order to carry out an entropy generation minimization at the system component level.
According to Kock and Herwing [33-34] and Herwing and Kock [35], the total entropy generation rate, $\dot{S}_{\text {gen }}$, is defined as the sum of entropy generation rate by direct dissipation, $\dot{S}_{D}$, entropy generation rate by indirect (turbulent) dissipation, $\dot{S}_{D^{\prime}}$, entropy generation by heat conduction due to mean temperature gradients, $\dot{S}_{C}$, and entropy generation by heat conduction due to fluctuating temperature gradients, $\dot{S}_{C^{\prime}}$ :

$$
\dot{S}_{g e n}=\dot{S}_{D}+\dot{S}_{D^{\prime}}+\dot{S}_{C}+\dot{S}_{C^{\prime}}
$$

The entropy generation terms are calculated using the following expressions [33-35]:

$$
\begin{gathered}
\dot{S}_{D}=\frac{\mu}{T}\left\{\begin{array}{c}
2\left[\left(\frac{\partial u}{\partial x}\right)^{2}+\left(\frac{\partial v}{\partial y}\right)^{2}\right] \\
+\left(\frac{\partial u}{\partial y}+\frac{\partial v}{\partial x}\right)^{2}
\end{array}\right\} \\
\dot{S}_{D^{\prime}}=\frac{\rho \varepsilon}{T} \\
\dot{S}_{C}=\frac{\lambda}{T^{2}}\left[\left(\frac{\partial T}{\partial x}\right)^{2}+\left(\frac{\partial T}{\partial y}\right)^{2}+\left(\frac{\partial T}{\partial z}\right)^{2}\right] \\
\dot{S}_{C^{\prime}}=\frac{\lambda_{t}}{T^{2}}\left[\left(\frac{\partial T}{\partial x}\right)^{2}+\left(\frac{\partial T}{\partial y}\right)^{2}+\left(\frac{\partial T}{\partial z}\right)^{2}\right]
\end{gathered}
$$

where $\varepsilon$ is the turbulence dissipation rate, and $\lambda_{t}$ is the turbulent thermal conductivity. They are defined by the turbulence model used.

The entropy generation is linked with the exergy destruction through the Gouy-Stodola equation:

$$
\Delta \dot{E}_{e x}=T_{0} \dot{S}_{g e n}
$$

where $\Delta \dot{E}_{e x}$ is the rate of exergy destruction and $\dot{S}_{g e n}$ is the entropy generation.

\subsection{Numerical procedure}

The equations presented above have been numerically solved using the commercial CFD code Ansys Fluent. SIMPLE algorithm has been selected. Continuity, momentum and the vapor phase transport equations have been descretized using second order upwind schemes. Due to the order of magnitude difference in phase's densities, under-relaxation factors have been adjusted.

The accuracy of numerical simulations has been assessed by comparing four mesh resolutions of 10,000 , $20,000,40,000$ and 60,000 cells for an inlet pressure of 2 $\mathrm{MPa}$ and an outlet pressure of 0.4 MPa using Schnerr and Sauer cavitation model. Results in terms of the mass flow rate ratio have been compared as shown in Table 2 . 
Table 1. Material properties

\begin{tabular}{lcc}
\hline & $\begin{array}{c}\text { Water } \\
\text { vapor }\end{array}$ & $\begin{array}{c}\text { Water } \\
\text { liquid }\end{array}$ \\
\hline Molecular weight, kg/kmol & \multicolumn{2}{c}{18.0} \\
Density, $\mathrm{kg} / \mathrm{m} 3$ & 0.5542 & 0.5542 \\
Heat capacity, J/kg.K & 2014 & 2014 \\
Dynamic viscosity, Pa.s & $1.34 \times 10^{-5}$ & $1.34 \times 10^{-5}$ \\
Thermal conductivity, W/m.K & 0.0261 & 0.0261 \\
\hline
\end{tabular}

The difference between mass flow rate ratios from mesh 3 and 4 do not exceeds $0.28 \%$. The numerical solution is independent of the mesh size by passing from 40,000 to 60,000 cells. Thus, it is judged that a mesh of 40,000 cells is sufficient and it is used for all runs.

Table 2. Mass flow rate ratio sensivity to mesh sizes

\begin{tabular}{lccc}
\hline Mesh & Cells number & $\dot{m}_{r}(\mathrm{~kg} / \mathrm{s})$ & $\delta(\%)$ \\
\hline 1 & 10,000 & 0.04600 & 1.440 \\
2 & 20,000 & 0.04629 & 0.607 \\
3 & 40,000 & 0.04642 & 0.280 \\
4 & 60,000 & 0.04654 & - \\
\hline
\end{tabular}

\section{Results and Discussions}

The discharge mass flow rates as function of the downstream pressure predicted using the three models have been compared to experimental measurements carried out by Ashrafizadeh and Ghassemi [26] as shown in Fig. 2. The discharge mass flow rate is expressed with a dimensionless form as

$$
\dot{m}_{r}=\frac{\dot{m}_{\text {actual }}}{\dot{m}_{\text {chocked }}}
$$

The downstream (exit) pressure is also expressed in a dimensionless form by

$$
p_{r}=p_{\text {out }} / p_{\text {in }}
$$

where $p_{\text {in }}$ is the upstream (inlet) pressure.

For all types of data, the flow presents two distinguished operating modes: the choked mode and the all-liquid mode. This trend is known as the characteristic curve of a cavitating venturi tube. At first sight, all numerical models tested allow a faithful reproduction of this characteristic curve. The mass flow rate ratio remains constant with the increase of the pressure ratio up to a critical value, from which it drops rapidly. The pressure ratio critical value is equal to 0.75 for experimental measurements. It is very close to 0.75 for numerical results according to the cavitation model used. There is general agreement in the literature that the critical value of the pressure ratio is around 0.8 [21]. Numerical results obtained using the Schnerr and Sauer model overestimate the mass flow ratio, especially in the chocked region where the maximum error is $4.2 \%$. It does not exceed $2.1 \%$ in the all-liquid region. This deviation can be attributed to both measurement errors and shortcomings of numerical models. The difference between cavitation models can be attributed to their different approaches in modeling the source term.

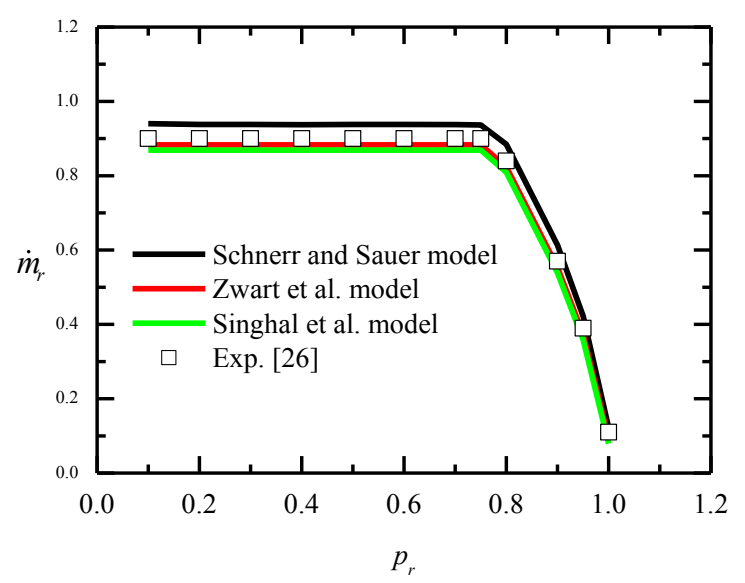

Fig. 2. Comparison of predicted mass flow rates from the three models at different pressure differentials $\left(\Delta p=p_{\text {in }}-p_{\text {out }}\right)$ with experimental measurements [26]

The assessment of cavitation models based on global venturi performance such as mass flow rate ratio is not sufficient. Comparisons should be made for local parameters such as velocity, pressure and vapor concentration. Results have been obtained for an inlet pressure of $2 \mathrm{MPa}$ and an outlet pressure of $1.4 \mathrm{MPa}$.

At the throat, static pressure drops below the saturated liquid pressure at the corresponding temperature causing the transition from liquid to vapor phase. This drop results from the acceleration of the fluid during its flow in the converging section of the cavitating venturi. According to Bernoulli's equation, the increase of the velocity involves a decrese in pressure. Starting from an inlet pressure of $2 \mathrm{MPa}$, the pressure decreases to reach a minimum pressure of $368.4 \mathrm{kPa}$ at the throat as shown in Fig. 3. Cavitation bubbles are created at the wall of the throat and separated from the leading edge of the throat. These bubbles move through the diffuser part and collapse into smaller bubbles and disappear at a specific length due to the pressure recovery at the diffuser part. Similar values are obtained by the three models.

Velocity prediction using the three models is depicted in Fig. 4. There is no significant difference in the velocity profiles obtained using the three models. Velocity contours confirm the previous results shown in Figure 3 . The flow is accelerated in the convergent part and the velocity reached the maximum values at the throat. By flowing towards the outlet of the venturi, the fluid loses its velocity. Through the throat part of the cavitating venturi, liquid is accelerated till the vapor pressure of water is reached.

The local entropy generation rate is maximal, as expected, near the wall due to friction between the fluid and the wall. The local entropy generation is directly related to the velocity distributions. Zones with large velocity gradients will naturally have high entropy generation due to friction as shown in Fig. 6. Far away from the venturi wall, where the velocity gradients are small, entropy generation is negligible. 


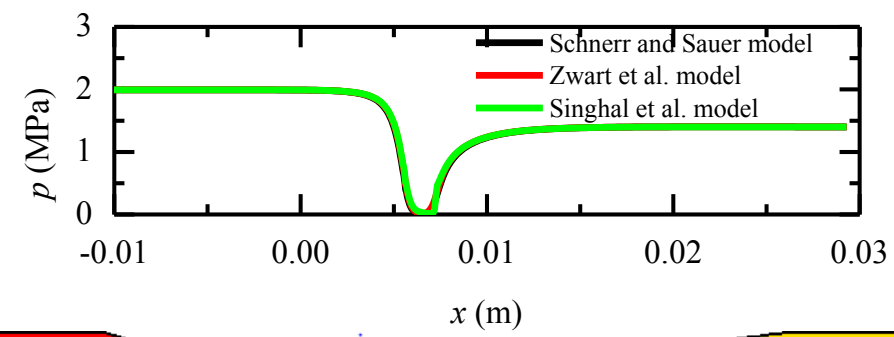

$a$.

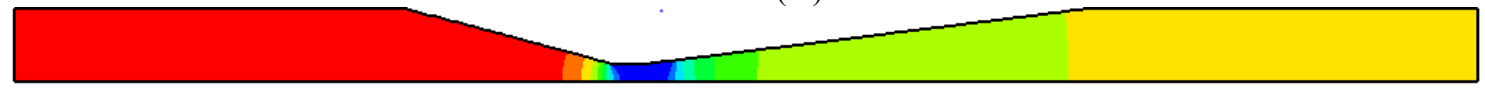

$b$.

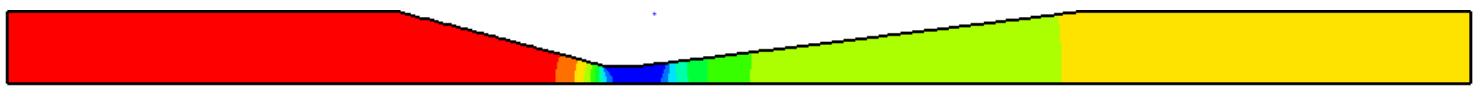

$c$.

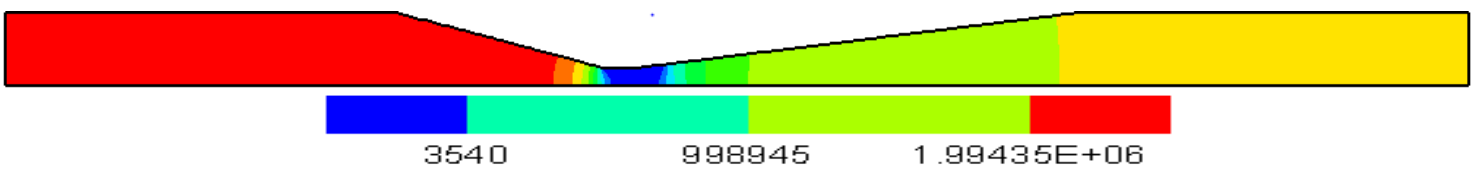

Fig. 3. Static pressure distribution within the cavitating venturi: $a$. Schenerr and Sauer model; $b$. Zwart-Gerber-Belamri model; $c$. Singhal et al. model

The exergy calculations have been performed using the return entropy generation values from the CFD analysis. Results, in terms of amount of exergy losses in the different regions of the flow domain in the cavitating venturi, are given in Fig.7. It appears that the main dominant exergy losses occur in the diverging part of the cavitating venturi. Indeed, in this part the flow undergoes high velocity gradient which produce higher values of entropy generated. The contributions of exergy losses in the divergimg part and the outlet duct are also significant. However, exergy losses in the inlet duct are negligible and those in the throat are smaller compared to the others parts of the cavitating venturi. These losses are mainly due to the large velocity and pressure gradients. An appropriate re-design of the regions with large exergy losses may contribute to potential enhancements.

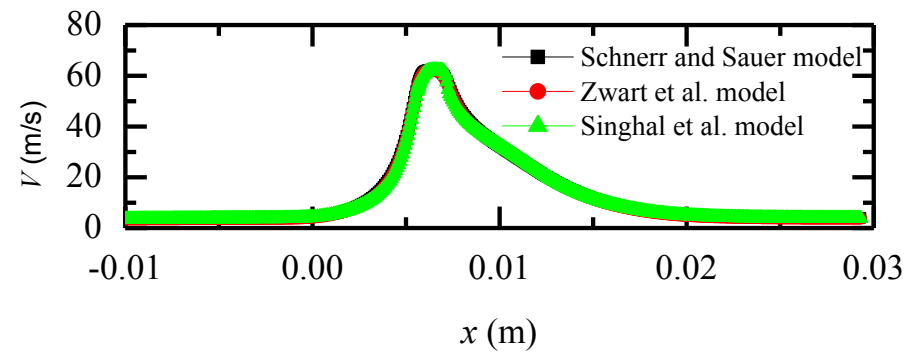

$a$

$b$.

c.

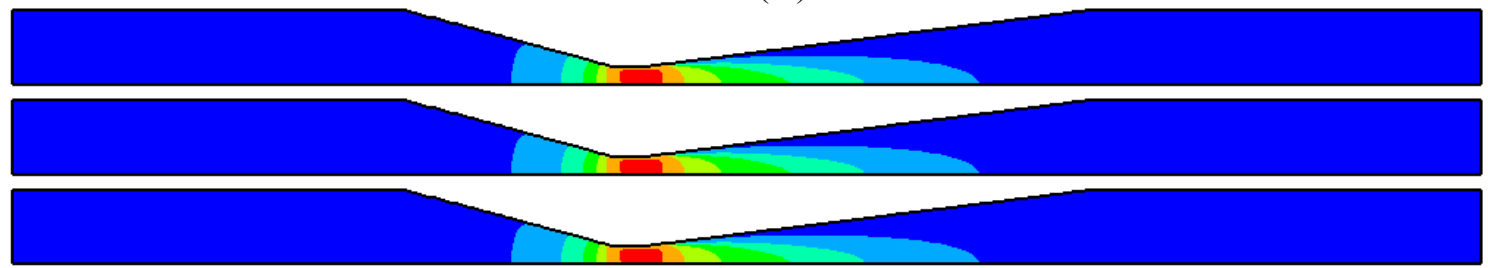

$\begin{array}{llllll}0 & 12.4596 & 24.9191 & 37.3787 & 49.8382 & 62.2978\end{array}$

Fig. 4. Velocity distribution within the cavitating venturi: $a$. Schenerr and Sauer model; $b$. Zwart-Gerber-Belamri model; $c$. Singhal et al. model

a.

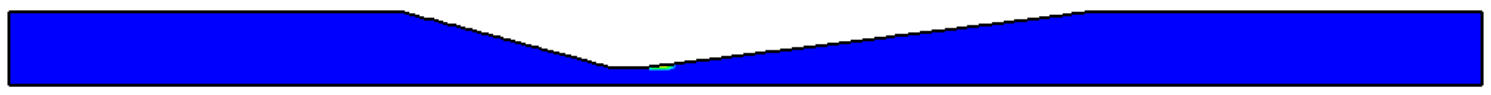

$b$.

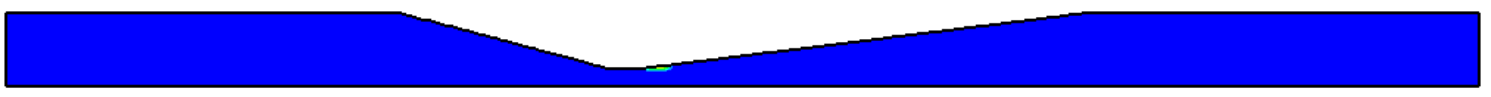

c.

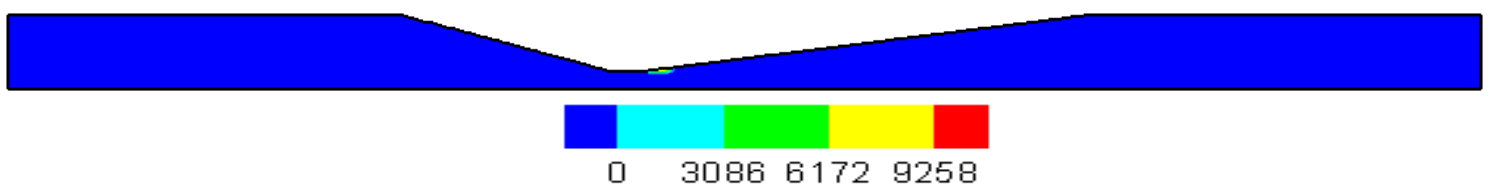

Fig. 5. Void fraction distribution within the cavitating venturi: $a$. Schenerr and Sauer model; $b$. Zwart-Gerber-Belamri model; $c$. Singhal et al. Model

\footnotetext{
* Corresponding author: ah ouadha@yahoo.fr
} 


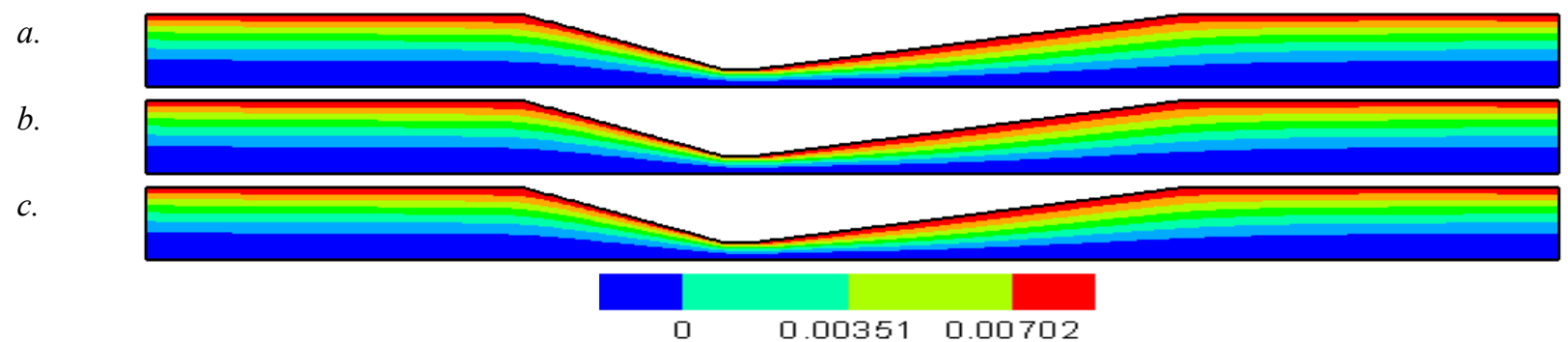

Fig. 6. Entropy generation distribution within the cavitating venturi: $a$. Schenerr and Sauer model; $b$. Zwart-Gerber-Belamri model; $c$. Singhal et al. model

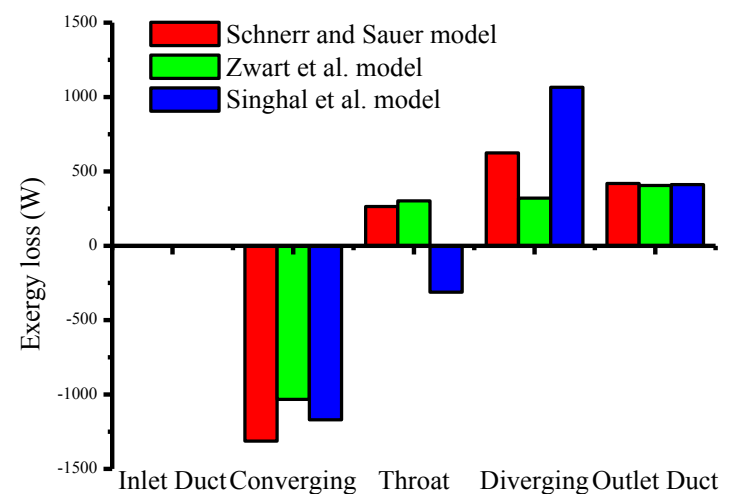

Fig. 7. Exergy losses within the cavitating venturi

\section{Conclusions}

A comprehensive assessment of three cavitation models, namely Singhal et al., Zwart-Gerber-Belamri and Schnerr and Sauer models in predicting the flow in a small size cavitating venturi tube has been performed. It is found that all models are capable of reproducing the physics of cavitation phenomena within the cavitating venturi. However, the Schnerr and Sauer model return higher values than the others model. It is also observed that most of the exergy losses occur in the converging and diverging parts of the venturi due to higher pressure and velocity gradients in these regions.

\section{References}

1. J. Ackeret, Technische Mechanik und Thermodynamik, 1 (1930)

2. F. Numachi, R. Kobayashi, S. Kamiyama, J. Basic Eng., 84 (1962)

3. F. Numachi, R. Kobayashi, Forsch. Ing.-Wes., 30, pp. 86-93. (1964)

4. J. A. Boure, A.A. Fritte, M.M. Gior, M. L. Reocreux, Int. J. Multiphase Flow, 3, (1974)

5. K. H. Ardon, R. B. Duffey, Int. J. Multiphase Flow, 4, (1978)

6. K. H. Ardon, Int. J. Multiphase Flow, 4, (1978)

7. N. T. Thang, M. R. Davis, Int. J. Multiphase Flow, 7, (1981)

8. J. H. Gummer, M. T. Thew, Proc. Instit. Mech. Eng., Par E: J. Proc. Mech. Eng., 212, (1998)

9. J. D. Sherwood, Int. J. Multiphase Flow, 26, (2000)
10. M. J. Gaston, J. A. Reizes, G. M. Evans, $14^{\text {th }}$ Australasian Fluid Mechanics Conference Adelaide University, Adelaide, Australia.( 2001)

11. H. H. Park, Y. Kim, E. Y. Jang, S.Y. Lee, J. Korean Soc. Aeronautical Space Sci., 30, (2002)

12. K. Sato, K. Hachine, Y. Saito, Proc. ASMEIJSME Joint Fluids Eng, 2, (2003)

13. G. Palau-Salvador, S. H. Frankel, $34^{\text {th }}$ AIAA Fluid Dynamics Conference and Exhibit, Portland, OR. (2004)

14. D. Kang, , K. Ahn, B. lim, S. Han, H. S. Choi, S. Seo, H. Kim, J. Korean Soc. Prop. Eng., 18, (2014)

15. B. Yazici, I. H. Tuncer, A. K. M. Ali, $3^{\text {rd }}$ Int. Conf. on Recent Adv. in Space Techno. 14-16 June, Istanbul, Turkey. (2007)

16. H. An, M. W. Plesniak, J. Fluids Eng., 130, (2008)

17. S. Barre, J. Rolland, G. Boitel, E. Goncalves, R.F. Patella, Euro. J. Mech. - B/Fluids, 28, (2009)

18. H. Sayyaadi, Fluid Dynamics Research, 42, (2010)

19. H. Ghassemi, H. F. Fasih, Flow Measu. Instru., 22, (2011)

20. J. Decaix, E. Goncalvès, Int. J. Heat Fluid Flow, 44, (2013)

21. M. Abdulaziz, Exp. Thermal Fluid Sci., 53, (2014)

22. M. G. Rodio, P. M. Congedo, Euro. J. Mech. B/Fluids, 44, (2014)

23. H. Tian, P. Zeng, N. Yu, G. Cai, Flow Measu. Instru., 38, (2014)

24. B. Charrière, J. Decaix, E. Goncalvès, Euro. J. Mech. B/Fluids, 49, (2015)

25. J. Mena, L. M. A. Ingle, V. Shirsat, A. Choudhuri, Flow Measu. Instru., 41, (2015)

26. S. M. Ashrafizadeh, H. Ghassemi, Flow Measu. Instru. 42, (2015)

27. X. Long, J. Zhang, J. Wang, M. Xu, Q. Lyu, B. Ji, Int. J. Multiphase Flow, 89, (2017)

28. J. Zhu, H. Xie, H. Feng, K. Zheng, X. S. Minqiang, Int. J. Heat Mass Transf., 112, (2017)

29. A. Bejan, Entropy generation through heat and fluid flow, New York: Wiley. (1982)

30. G. H. Schnerr, J. Sauer, $4^{\text {th }}$ Int. Conf. Multiphase Flow, New Orleans, USA. (2001)

31. P.J. Zwart, G. Gerber, T. Belamri, $5^{\text {th }}$ Int. Conf. Multiphase Flow (ICMF'04). (2004) 
32. K. Singhal, M. M. Athavale, H. Li, Y. Jiang, ASME J. of Fluids Eng., 124, (2002)

33. F. Kock, H. Herwig, Int. J. Heat Mass Transf., 47, (2004)

34. F. Kock, H. Herwig, Int. J. Heat Fluid Flow, 26, (2005)

35. H. Herwig, F. Kock, Heat Mass Transfer, 43, (2006) 